\title{
Protée
}

\section{Dérivations de l'allégorie dans la photographie contemporaine}

\section{Mirelle Thijsen}

Volume 33, numéro 1, printemps 2005

L’allégorie visuelle

URI : https://id.erudit.org/iderudit/012266ar

DOI : https://doi.org/10.7202/012266ar

Aller au sommaire du numéro

Éditeur(s)

Département des arts et lettres - Université du Québec à Chicoutimi

\section{ISSN}

0300-3523 (imprimé)

1708-2307 (numérique)

Découvrir la revue

\section{Citer cet article}

Thijsen, M. (2005). Dérivations de l'allégorie dans la photographie contemporaine. Protée, 33(1), 49-57. https://doi.org/10.7202/012266ar

\section{Résumé de l'article}

Quelle est la place occupée par l'allégorie dans la photographie contemporaine ? L'utilisation du mode allégorique marque, depuis le début de ce siècle nouveau, un tournant décisif. Les pratiques photographiques actuelles s'imprègnent de dérivations allégoriques : de nombreux artistes photographes, des femmes surtout, majoritairement britanniques, font ouvertement usage de l'allégorie dans leurs oeuvres, assurant ainsi à la suite des travaux des générations d'artistes photographes des années 1980 et 1990 (Evergon, Sherman, Knorr, Buckland, Richon, Cohen, Leriche, etc.) la survie de ce mode " académique ». En cela ils s'appuient sur des conventions de représentation et de figuration héritées de la tradition de l'art occidental, fondées donc sur une esthétique bien établie.

Alors que l'on dispose de nombreuses études qui alignent l'allégorie sur les productions du théâtre, de la littérature, de la sculpture, de la peinture et du cinéma, on s'est très peu intéressé d'un point de vue sémiotique aux stratégies et aux méthodes de travail des artistes qui adoptent le mode allégorique, et partant aux discours critiques qui leur sont consacrés. C'est là la préoccupation principale du présent article. On cherchera donc à dégager la très grande variété d'outils empruntés par des artistes impliqués à fond dans des projets esthétiques très divers et souscrivant à des positions politiques différentes, et à étudier la manière dont ils génèrent dans l'imagerie fixe des effets narratifs et le sens allégorique. Il sera également opportun de mettre en évidence certains thèmes récurrents, liés à la culture, aux institutions, au pouvoir, à la diversité sexuelle, et la manière dont ces artistes ont su dépasser et étendre les codes de représentation classiques.
Ce document est protégé par la loi sur le droit d'auteur. L'utilisation des services d’Érudit (y compris la reproduction) est assujettie à sa politique d'utilisation que vous pouvez consulter en ligne.

https://apropos.erudit.org/fr/usagers/politique-dutilisation/ 


\section{DÉRIVATIO N S DE L'A LLÉG O RIE DANS LA PHOTO GRAPHIE CONTEM PO RAINE}

M IRELLE THIJSEN

Traduit de l'anglais par Tony Jappy

Quelle est la place occupée par l'allégorie dans la photographie contemporaine? L'utilisation du mode allégorique marque, depuis le début de ce siècle nouveau, un tournant décisif. Les pratiques photographiques actuelles s'imprègnent de dérivations allégoriques: de nombreux artistes photographes, des femmes surtout, majoritairement britanniques, font ouvertement usage de l'allégorie dans leur œuvre (Sarah Jones, Melanie Manchot, Sam Taylor-Wood, Yasumasa Morimura, Pierre et Gilles et John Goto) assurant ainsi, à la suite des travaux des générations d'artistes photographes des années 1980 et 1990 (Jeff Wall, Sorel Cohen, Evergon, Karen Knorr, David Buckland, Olivier Richon, Dany Leriche, Cindy Sherman, etc.), la survie de ce mode «académique». C'est ainsi que le passé, le présent et le futur s'intègrent dans l'œuvre photographique et que la temporalité joue sur les diverses lectures que nous en faisons.

Les artistes photographes qui font appel au mode allégorique se fondent sur des conventions picturales et des conventions de figuration communes à l'art occidental. De la même façon, le contenu allégorique de leur art relève d'une esthétique bien établie, et la stratégie artistique qui consiste à s'approprier les chefs-d'œuvre de l'histoire de l'art s'appuie elle aussi sur une longue tradition et reste encore un thème incontournable dans la mise en place d'expositions ${ }^{1}$. C'est qu'en situant délibérément ainsi leur œuvre dans la mémoire collective, ils font appel à ce matériau référentiel dans la construction sociale de la compétence visuelle.

Le présent article voudrait examiner de plus près la notion d'allégorie dans les pratiques photographiques contemporaines et, par la même occasion, sa place dans le discours de la critique de l'art. Alors que le rapport entre l'allégorie photographique contemporaine et les productions du théâtre, de la littérature, de la sculpture, de la peinture et du cinéma a souvent été remarqué, peu d'études ont porté spécifiquement sur la stratégie artistique et sur les méthodes de travail des artistes photographes qui travaillent principalement dans un cadre allégorique. 


\section{QUELQUES CARACTÉRISTIQUES DE L'ALLÉGORIE PHOTOGRAPHIQUE CONTEMPORAINE}

L'utilisation faite aussi bien de l'allégorie que de la métaphore dans les actuelles pratiques photographiques éclectiques demande à être approfondie. Nous chercherons à dégager la très grande variété d'outils empruntés par des artistes qui sont engagés à fond dans des projets esthétiques très divers et qui souscrivent à des positions politiques différentes, et à étudier la manière dont ils génèrent, dans l'imagerie fixe, des effets narratifs et le sens allégorique. Nous nous intéresserons donc à la communauté des outils employés, notamment, et à la manière dont leurs références coïncident et se renvoient les unes aux autres. Alors que certains artistes photographes visent à reproduire les origines de l'art, d'autres se construisent un langage visuel emprunté aux images stylisées de notre culture de consommation: l'allégorie du quotidien, en quelque sorte. Juxtaposition de thèmes, représentation visuelle du pouvoir, divers modèles $\mathrm{du}$ comportement social ainsi que des rôles sociaux conflictuels, voilà quelques références de leur problématique commune, à partir de laquelle ils concentrent les rapports qui existent ou qui sont à établir entre image et texte et, dans des œuvres "mesurées et posées", opposent l'ironie à la nostalgie. Il ne faut pas oublier dans ce type d'œuvre, bien sûr, la part d'ambiguité (l'allégorie en tant que moralité et l'allégorie dans son rôle de commentaire des normes sociales et politiques sont ambiguës au même degré), le non conventionnel et la prolifération des identités.

Par conséquent, on peut recenser en vrac les caractéristiques principales du genre: mise en scène "chorégraphiée», potentiel narratif, représentation figurale, jeu dramatique, niveaux d'interprétation multiples, temporalité, statut ambigu de l'imagerie, sérialité et qualité formaliste. Le genre est donc figuratif, faisant une large part à la couleur, simple technique servant à esthétiser et à dramatiser l'abject.

Commenter la persistance de l'allégorie dans la photographie contemporaine revient à approfondir le rapport entre la photographie, l'objet et la représentation, la rhétorique et l'esthétique de l'iconographie allégorique, à porter un regard sur la culture, les institutions culturelles, le pouvoir, les conditions de la vie sociale, la diversité sexuelle. Car la variété des formes et des genres se manifeste dans la diversité des pratiques consacrées à un mode bien établi dans l'histoire de l'art. C'est ainsi, par exemple, que la majorité des artistes photographes femmes qui font appel au mode allégorique, notamment Cindy Sherman, Karen Knorr, Melanie Manchot, Sorel Cohen, Dany Leriche, remettent en question la représentation, dans l'iconographie occidentale, du corps féminin.

Dans une étude fondamentale, Craig Owens retrace clairement le regain d'influence que connaît l'allégorie dans notre culture contemporaine. Partant de la connotation négative associée à l'allégorie du fait que les allégoristes se sont longtemps contentés de reproduire et de s'approprier les images d'autrui, se servant en somme de l'allégorie comme simple ornement rhétorique afin de moraliser l'image, Owens montre comment on a longtemps considéré l'allégoriste comme historiciste, antithèse du réaliste ou du moderniste (1984: 205). Mais, grâce à «[ses techniques d']appropriation, sa spécificité situationnelle [site specificity], son impermanence, sa capacité d'accumulation, sa discursivité et son hybridation, en plus de son éclectisme et de sa qualité statique» (ibid.:206), la photographie constitue, aux yeux d'Owens, le médium allégorique par excellence, associant le potentiel allégorique de la photographie principalement à des artistes postmodernes qui portent un regard critique sur la tradition documentaire, notamment Sherrie Levine et Robert Longo. En revanche, depuis les années 1980, les tableaux allégoriques conçus par Karen Knorr, Olivier Richon et, à un degré moindre, Bernhard Prinz présentent une structure plus lourdement et plus explicitement narrative, où s'intègrent divers éléments linguistiques, souvent conçus (chez Prinz, notamment) comme des assertions iconisées. Selon Owens, les rapports de réciprocité informant texte et image dans les allégories photographiques méritent une plus grande attention (ibid.: 208). 
Depuis le milieu des années 1990, les œuvres d'art photographiques à caractère allégorique sont devenues des images muettes, rendant ainsi "glissante» leur visée narrative (Soutter, 2000) et donnant au titre des séries d'œuvres, et du même coup à celui de chaque œuvre à l'intérieur de la série, une importance accrue, car un titre est porteur de références spécifiques à l'histoire de l'art, à la philosophie, à la psychanalyse, à la littérature et au social. Les documents discutés par Soutter font partie d'une série plus grande et plus variée d'œuvres photographiques allégoriques, donc qui sont à plus grand pouvoir de contextualisation. Ces séries complémentaires renvoient également à la sérialité «artistique» dans la culture de masse (film, bande dessinée, roman-photo, etc.). De telles œuvres conservent, dans des séquences non linéaires, de courtes trajectoires narratives, offrant ainsi à l'observateur une structuration et la «possibilité de configuration» (Barthes, 1973) «grâce aux procédés de la sélection et de l'organisation» et du «réalisme performatif» (Slyce, 1999).

\section{ALLÉGORIE OU CINÉMATOGRAPHIE?}

Dans la photographie contemporaine, l'allégorie est une construction fictive qui implique une part de vérité dans la mesure où elle intègre des fragments du réel, comme dans un film documentaire (Slyce, 1999; Soutter, 2000; Dercon, 2000), et où l'on trouve de surcroît des références explicites à une planification à caractère documentaire ${ }^{2}$. Comme dans le cas des productions cinématographiques professionnelles, des artistes photographes comme Jeff Wall, Karen Knorr, Sam Taylor-Wood, David Buckland et Sarah Jones établissent des scénarios complets avant d'entreprendre les prises de vue.

Ils opèrent en réalisateurs de films, en petites entreprises de production, dont l'équipe construit la scène, où les costumes sont conçus, où les aspects techniques et les problèmes de composition sont organisés et surveillés. Mais si l'œuvre de Goto, de Jones ou de Wall est franchement cinématographique, les citations qu'on y trouve concernant l'histoire du cinéma, de la photographie et de la peinture sont néanmoins accessoires et d'importance secondaire. L'esthétique des ces photographes se fonde en partie sur les grandes manifestations de l'histoire de l'art, à laquelle il faut ajouter des composantes sans existence propre qui constituent l'élément fictif. Il leur arrive parfois de s'approprier une image existante qui présente un sujet traité par un autre artiste ou qui reprend un problème d'ordre sociopolitique ou philosophique. Dans la plupart des cas, il en résulte une image singulière constituée d'une constellation artificielle d'éléments, imprégnée de suspens, d'une réunion de moments disparates.

Le milieu des années 1960 a connu un mouvement d'expérimentation visant à assimiler la photographie à la peinture. Beaucoup de photographes ont opté pour un format plus grand et se sont servis de techniques et de technologies nouvelles empruntées au monde de la publicité et du design. Avec l'introduction de l'informatique, les frontières du médium ont été repoussées encore plus loin, ouvrant au mode allégorique des possibilités d'invention apparemment sans limites. De plus, le caractère artificiel de l'image photographique est devenu quasiment invisible. L'œuvre Dead Troops Talk (1986) de Jeff Wall, par exemple, est à la fois une mise en scène extraordinairement cinématographique et un photomontage dont l'assemblage d'origine est indétectable. Ses photographies numérisées se composent de nombreux clichés individuels et se présentent comme des transparents à grand format disposés dans des vitrines éclairées par l'arrière. (

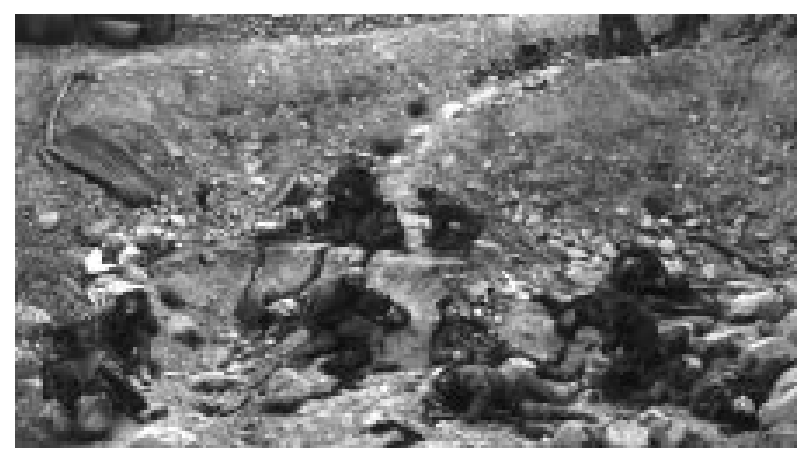

Planche 1. Jeff Wall, Dead Troops Talk (A Vision After an Ambush of a Red Army Patrol, near Moqor), Afghanistan, hiver 1986. 
cause de la technologie cibachrome utilisée, l'esthétique et la beauté des ces images ont été jugées simplement «matérialistes» [Atkinson, 1993: 29].)

De cette manière, fort de l'influence conjointe de l'histoire de l'art et du cinéma qu'il a subie, Wall associe à des stratégies documentaires des techniques et des mises en scène empruntées au monde du théâtre et au domaine de la publicité.

\section{CATAlOgue DES TYPES}

Jusqu'à quel point s'agit-il de portraits historiques? Et quels en sont les prototypes? Le décorateur professionnel britannique David Buckland a conçu, au cours des années 1980, de grands tableaux typologiques au moyen de la technique de la projection frontale (The Numerologist, 1984; The New Puritan, 1985; The Commissioner, 1987), mais son œuvre la plus ambitieuse reste The Marriage of Arnolfini (Re-viewed) (After Jan van Eyck, 1986). Des objets emblématiques sont disposés avec artifice, alors que des photogrammes, fonctionnant comme décor, et des accessoires de théâtre servent à définir le sujet et à relier passé et présent. Le profil qui informe le Numerologist est emprunté au célèbre portrait du Comte d'Urbino de Piero della Francesca et se trouve collé sur une image de la ligne des toits de la Cité de Londres d'aujourd'hui, vue de la Tamise, afin de replacer le sujet, chorégraphe de renom, dans son milieu professionnel.

De telles œuvres relèvent d'une longue tradition. Dans le tableau de Gustave Courbet L'Atelier $d u$ peintre (1854-55), au sous-titre provocateur: «Allégorie réelle déterminant une phase de sept années de ma vie artistique", nous trouvons représentés, en plus des amis du peintre, des "types" généraux plutôt que des individus reconnaissables (notamment le vétéran, le chasseur, le Juif) (Fried, 1981: 94). Transformer l'allégorie en un catalogue de types, c'est là une stratégie adoptée par un nombre croissant d'artistes photographes: David Buckland, Bernhard Prinz, Cindy Sherman, Yasumasa Morimura et, à un degré moindre, Pierre et Gilles. Ceux-ci, deux artistes français, incorporent le mode allégorique dans une

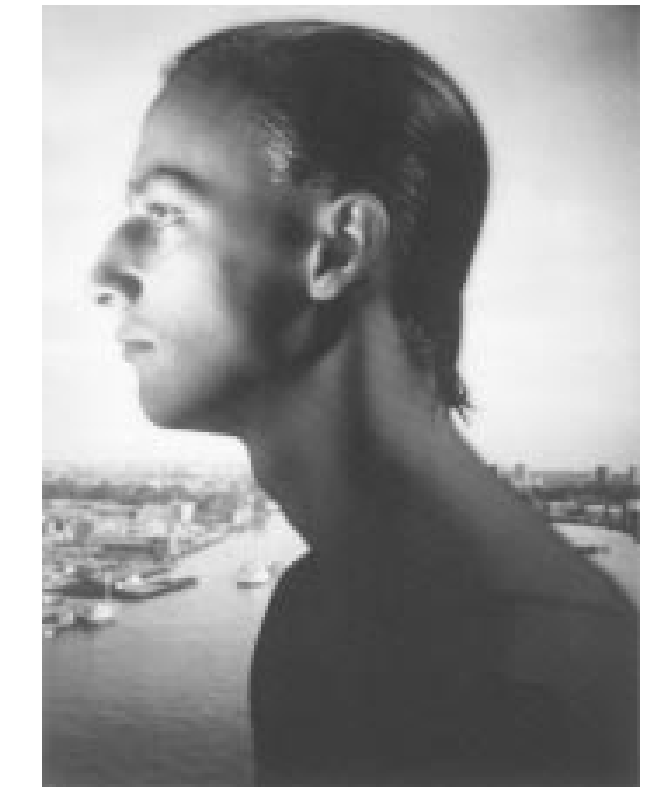

Planche 2. David Buckland, The Numerologist, 1984. Matthew Hawkins. (c) David Buckland, 2005.

imagerie commerciale issue du monde de la publicité. Ce potentiel d'implication mutuelle du commerce et de l'art est personnifié sous l'aspect de galeristes, chorégraphes, stylistes, commissaires, acteurs, mannequins, etc., aussi bien dans l'œuvre de David Buckland que dans celle de Pierre et Gilles, à ceci près que ces derniers fabriquent des images hybrides qui s'intègrent d'ores et déjà dans la culture visuelle de masse, transformant en icônes des saints, des personnages mythologiques et des idoles.

Dans une série de photographies retouchées à la peinture (Pierre prend les photos, Gilles applique la peinture acrylique au pinceau), ils ont conçu, pour un monde imaginaire et nickel de dandy, des décors kitsch et maniérés en carton, un monde d'accessoires, de costumes, de plateaux, de maquillages, de performances, un monde de sentiments où l'on pleure des larmes de glycérine. Ici, en retravaillant le réel, rien de laid. À la différence de leur prédécesseur dans ce domaine, l'artiste Steven Arnold de Los Angeles, que leurs photographies rappellent, Pierre et Gilles empruntent rarement le mode allégorique, car ils ne représentent guère des concepts sous la forme de personnages définis par des attributs. Le Purgatoire (1990) est une exception. 


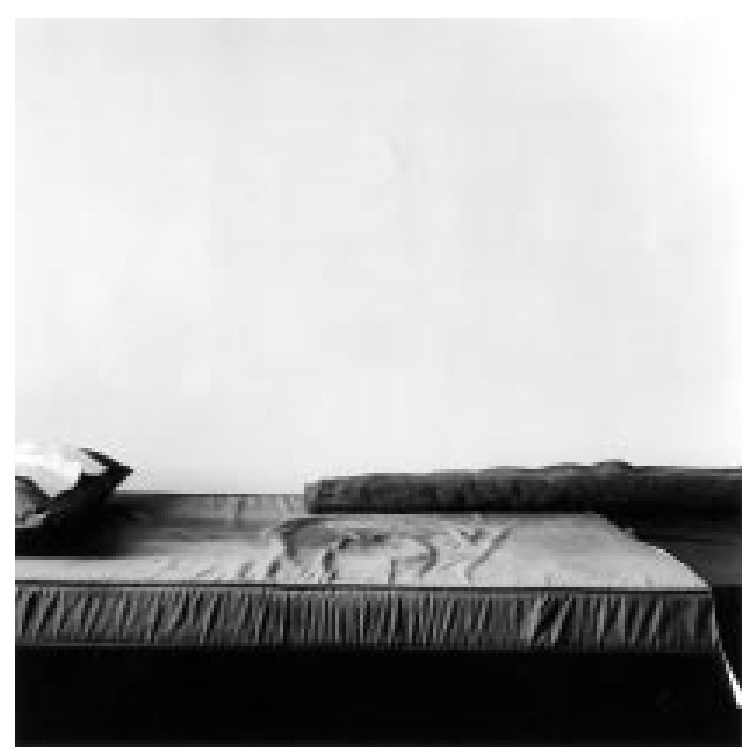

Planche 3. Sarah Jones, Consulting Room [couch] [XIX], 1998. Courtesy Maureen Paley.

\section{LE PORTRAIT ALLÉGORIQUE}

La mise en scène, soit d'autrui, soit du photographe lui-même, est un autre trait distinctif de l'allégorie photographique contemporaine. Il en résulte des images illustrant un style personnel, qui permettent à l'observateur de les regarder dans un contexte neutre, libre de toute référence biographique. Ces portraits dépouillés représentent paradoxalement une absence d'identité et de personnalité, et la contextualisation des gestes, qui caractérisent ces images en gros plan, renvoie à une identité sociale plus large.

Courbet a fait preuve d'une extraordinaire prédilection pour le portrait, produisant un nombre d'autoportraits ambitieux qui exhibent une proximité physique extrême (Fried, 1981: 99), choix qui, tout comme la pose frontale, constitue l'une des caractéristiques majeures du portrait allégorique. C'est ainsi que certaines ouvres photographiques contemporaines représentent des acteurs ou des personnes recrutées au hasard des rencontres quotidiennes - parfois un piquant mélange d'acteurs et d'amis de l'artiste (Bernhard Prinz, Pierre et Gilles, Sarah Jones, Karen Knorr), parfois les artistes euxmêmes se mettant en scène (Cindy Sherman, Yasumasa Morimura, Sam Taylor-Wood) -, qui regardent tous fixement l'appareil ou, selon Slyce (1999), qui communiquent le regard. Il manque à ces portraits allégoriques l'expression d'un état d'esprit précis, l'artiste cherchant autant que possible la neutralité; il en résulte une œuvre photographique à l'image du sujet: distant, parfait, précis.

Prinz et Jones préfèrent mettre en scène des adolescents, beaux pour la plupart, alors que Sherman et Morimura se projettent comme des icônes dans des autoportraits qui tirent grand parti de déguisements, de prothèses diverses, de masques et d'accoutrements bizarres, servant à représenter de jeunes mariées, des princesses, des anges, des allégories moralisantes. Par exemple, l'artiste japonaise postmoderne Morimura se photographie sous la forme de la naine disgracieuse des Ménines de Vélasquez (1656) ou comme une Judith ou une Marlène Dietrich aux yeux bridés.

Sherman pose en nostalgique cynique sous les traits d'un clerc médiéval mal fagoté ou d'un personnage huppé de la Renaissance. Ce goût du difforme renvoie à l'expérience humaine prise dans la réalité dure de la vie quotidienne. C'est ainsi que le grotesque et le mode allégorique se trouvent confrontés dans l'œuvre photographique de Morimura et de Cindy Sherman. De la même façon, comme le note Jean-François Chevrier qui y voit un maniérisme grotesque et un humour noir, l'œuvre de Jeff Wall associe mode allégorique et événements historiques. Enfin, tout comme Sherman, Morimura et Wall, John Goto cherche lui aussi à réintroduire dans la culture de l'art photographique contemporain la satire et l'humour.

Pierre et Gilles, pour leur part, mettent en scène des icônes de la jeunesse, tirant parti dans la plupart des cas d'une reconstruction de l'idéal, de l'extravagant, de la représentation de la puissance dans un monde profane fondé sur des références classiques, voulant ainsi engager le dialogue entre la photographie contemporaine et les canons de l'histoire de l'art. De plus, les poses adoptées dans ces portraits allégoriques - de proportions souvent monumentales - oscillent entre l'allégorie complexe et le portrait statique mais souvent répété, dans lequel 


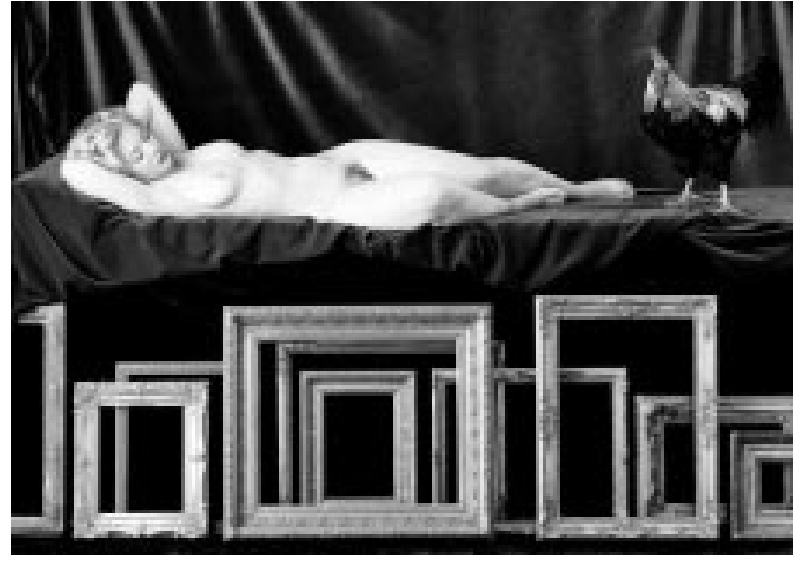

Planche 4. Dany Leriche, Virilité, 2000.

sont représentés par leurs attributs spécifiques le vice et la vertu (Bernhard Prinz: Stratégie, Liste) ou des concepts plus universels (Dany Leriche: La Philosophie, Le Destin). Dans la plupart des cas, les artistes font grand usage de personnes réelles, ce qui permet d'élargir leur signification éthique, sociale et culturelle et de mettre en avant le caractère artificiel de la mise en scène. C'est ainsi que le portrait allégorique possède une sorte d'objectivité froide: il constitue une sorte d'inventaire des types humains. À ce propos, on notera que Richon, Knorr et l'artiste photographe parisienne, Dany Leriche, ont beaucoup intégré dans leur œuvre des références tant politiques que philosophiques et encyclopédiques. Depuis 1988, par exemple, Dany Leriche traduit dans l'idiome moderne de l'allégorie photographique l'iconologie de Cesare Ripa $^{3}$. Son œuvre Les Filles de Ripa met en scène des femmes nues de tous âges, qui personnifient ainsi «la fraude» ou «le doute» (les personnifications allégoriques représentent le plus souvent des femmes). De cette façon, et en collaboration avec des scientifiques, des artistes et des philosophes qui commentent les thèmes de Ripa et qui proposent la définition de notions abstraites, Leriche est en train de constituer un dictionnaire iconologique du monde contemporain. Virilité, par exemple, montre une jeune femme nue, voluptueuse, étendue sur un lit de velours rouge, un coq à ses pieds. Une série de cadres dorés mais vides, posés autour du lit, renvoie à l'histoire de la représentation.

\section{ALLÉGORIE DU QUOTIDIEN}

L'œuvre de l'artiste photographe londonienne, Sam Taylor-Wood (film, vidéo, sculpture, photographie), comme celle de Sarah Jones (photographie) sont éclectiques; elles renvoient à la mémoire iconographique et aux codes de l'histoire de l'art, ouvrant ainsi à un public plus large le concept même d'allégorie. Elles emploient dans leurs recherches des images célèbres puisées dans le vaste domaine de la culture visuelle; c'est ainsi que l'iconographie classique intègre le quotidien contemporain et le champ du réel. Cette transposition de la scène religieuse à l'allégorie du quotidien contemporain est un développement récent, et beaucoup d'artistes contemporains travaillent dans un rapport plus ou moins précis avec ces matrices culturelles, se servant d'accessoires de la vie courante et en soumettant l'iconographie conventionnelle à des innovations formelles, afin de créer «de nouvelles iconographies et des manières de voir plus flexibles» (Santacatterina, 2002: 53). Les photos installations des deux artistes sont des représentations de plateaux de cinéma, images attirantes qui offrent un lien direct au monde réel et au vernaculaire.

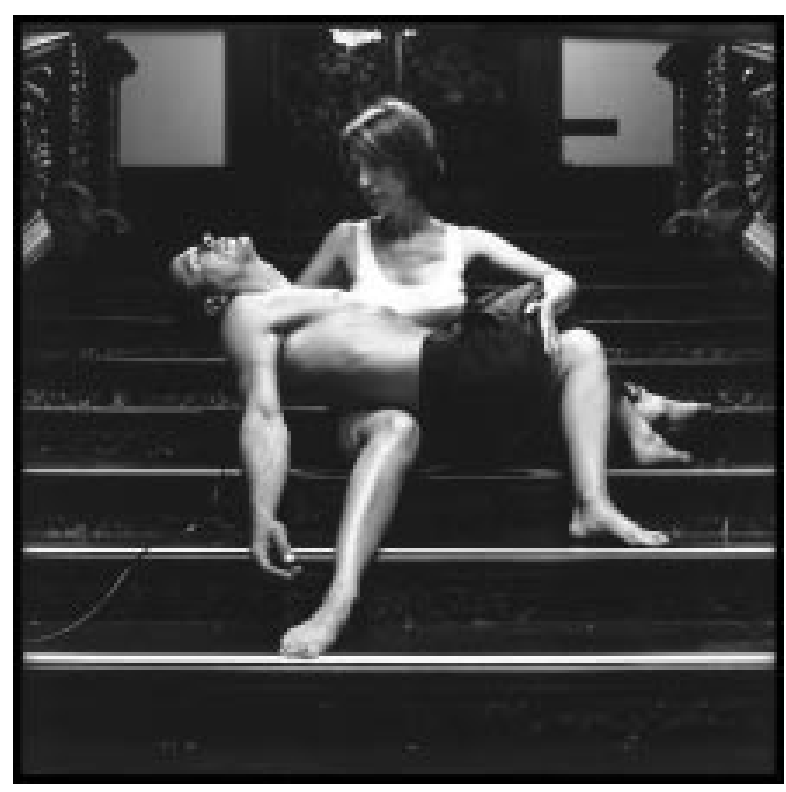

Planche 5. Sam Taylor-Wood, Pietà, $2001^{4}$. Film 35mm/DVD, 1 min 57 s. (c) Sam Taylor-Wood. Courtesy Jay Jopling/White Cube (Londres). 


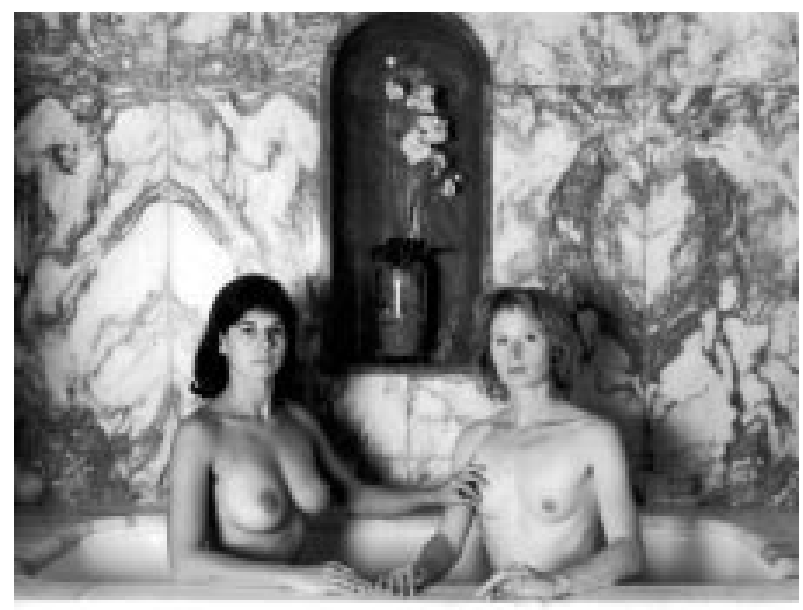

Planche 6. Melanie Manchot, The Fontainebleau Series, Emma \& Charlie, $2001^{5}$. (C) Melanie Manchot, 2005.

En particulier, c'est cette iconographie du quotidien, salles de bains, salles de séjour, paysages urbains, qui imprègne La Série Fontainebleau (2001) de Melanie Manchot. La photographe établit le rapport entre cette série photographique et le tableau original du XVI siècle au moyen d'un jeu de codes et de conventions (titre, baignoire, les deux femmes nues, les gestes). C'est ainsi que les œuvres photographiques et le tableau problématisent la place de la sexualité féminine à l'intérieur de l'histoire de la représentation artistique.

Mais alors que la structure iconique du tableau est contrainte par le cadre, les photographies, quant à elles, exhibent ouvertement leur rapport au réel: couleur de la peau, tâches de vin, poils de l'aisselle, autant de traits qui proclament le caractère impitoyable du médium photographique. Afin d'approfondir dans leurs allégories l'illusion du vernaculaire et l'illusion rétrospective de l'histoire, plusieurs artistes photographes (Goto, Prinz, Wall) ont adopté la technologie numérique. En particulier, certaines des œuvres de Goto et de Wall, qui reposent sur les genres de l'histoire de la peinture, ressemblent à d'anciens paysages, comme des tableaux vivants: des scènes chorégraphiées qui fonctionnent à des niveaux multiples. Les «tableaux photographiques» de Wall (Chevrier, 1995) sont construits, mis en scène pour ensuite être modifiés numériquement afin d'explorer une gamme très large de thèmes sociopolitiques (violence, racisme, sexe, identité, histoire, représentation). Ces problématiques sont allégorisées grâce à la manipulation numérique empruntée à la publicité et aux stratégies de marketing du capitalisme tardif. En même temps, ses tableaux hybrides entretiennent un dialogue avec certains peintres du $\mathrm{XVIII}^{\mathrm{e}}$ et XIX ${ }^{\mathrm{e}}$ siècles, par exemple Eugène Delacroix et Théodore Géricault. Chevrier définit le «tableau photographique», décrit en détail ses aspects formels et montre comment Jeff Wall a su, grâce aux procédés numériques, en transformer le mode pictural en portant un jugement sur la positon dialectique de la photographie entre les beaux-arts et les médias ${ }^{6}$. Dead Troops Talk est l'allégorie de la mort de l'Armée Rouge, ce qui en fait un "tableau d'histoire» - encore un terme de Chevrier (ibid.: 12) -, dont les «ingrédients» sont le capitalisme d'entreprise, le drame historique, l'idéologie occidentale et la pédagogie marxiste (Atkinson, 1993: 29).

De la même façon, Eco Warriors (2000-2001), de la série High Summer de John Goto, est un mélange virtuel de jardins anglais du XVIII siècle et du paysage arcadique français du XVII e siècle. Un petit paysage typiquement anglais se trouve doté d'une signifiance métaphorique et historique plus large. On y trouve associés des personnages caractéristiques de la région et des types humains numérisés, chaque groupe occupant des zones d'un paysage de parc artificiel et méticuleusement entretenu avec, comme arrière-plan, une architecture néoclassique. Grâce à ces traitements numériques, ce qui était à l'origine un paysage simple

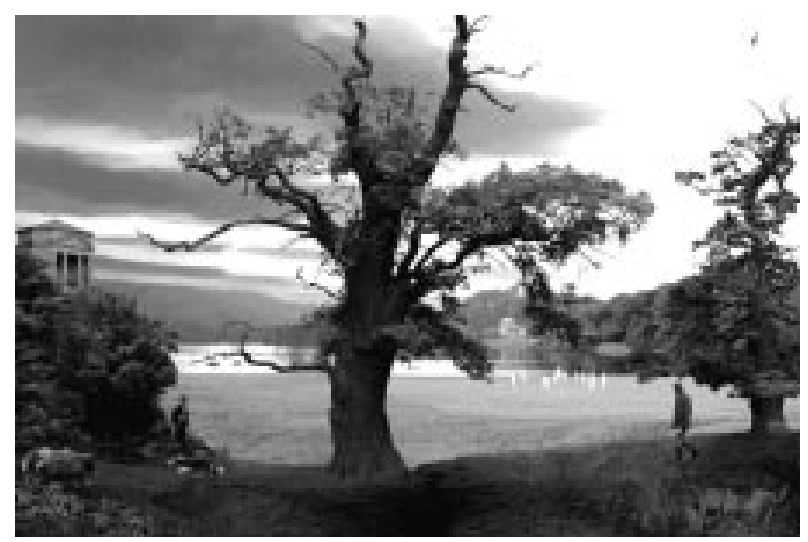

Planche 7. John Goto, Eco Warriors, 2000-2001. (C) John Goto, 2005. 
est transformé en un théâtre en plein air fascinant, aussi bien dans l'œuvre de Goto que de Wall: les protagonistes ont été empruntés à des situations réelles, de même que des artefacts culturels obtenus par manipulations numériques sont positionnés selon les règles de composition de la représentation traditionnelle.

\section{CONCLUSION}

L'allégorie est un mode institutionnalisé de l'histoire de l'art, elle est formée, modelée pour ainsi dire par l'histoire, de nature extravagante (Owens, 1980: 215); et, en général, les stratégies artistiques qui visent la "construction et la déconstruction des représentations culturelles» (Wallis, 1987: xiv) sont immanquablement allégoriques. Les photographes qui travaillent sur le mode allégorique sont constamment en train de repousser plus loin les codes classiques de la représentation. Mais, en même temps, le potentiel de lecture narrative est compromis. Autrement dit, les lectures allégoriques associées à la photographie contemporaine augmentent la multivalence des images, les rendant moins explicites sur le plan sémiotique.

Par ailleurs, la plupart des représentants de cette nouvelle photographie se considèrent davantage «artistes» que "photographes», bien que presque tous travaillent exclusivement avec le médium photographique; et nombreux sont ceux dont l'ambition de devenir célèbres est à peine cachée dans leurs œuvres. Par exemple, Wall, Sherman, Knorr, Prinz ont tous contribué à rétablir le mode allégorique et avaient déjà acquis la notoriété au moment où la photographie a commencé à dominer le monde de l'art et le marché de l'art qui le sous-tend. La photographie a ainsi fait de plus en plus partie, selon l'expression de Sekula (1990), d'«une culture d'entrepreneurs» aux dépens du potentiel critique du médium et de son agenda politique ${ }^{7}$. Au moment où les frontières entre art, photographie et culture de consommation sont tombées, le mode allégorique adopté par la photographie contemporaine reste l'expression d'une culture élitiste. Et bien que les artistes photographes partagent les mêmes outils et les mêmes références, l'allégorie photographique contemporaine n'a pas une signification unifiée: ses concepts évoluent sans cesse pour être intégrés à des stratégies artistiques nouvelles et aux discours changeants de la critique de l'art.

\section{N O TES}

1. Voir, par exemple, les catalogues qui portent spécifiquement sur l'appropriation des chefs-d'œuvre de la tradition occidentale: Perspektief, no31-32, 1989; Quotation: Re-presenting History, Winnipeg (Winnipeg Art Gallery), 1994; Los Géneros de la Pintura. Una visión actual, Las Palmas (Grande Canarie) (Centro Atlantico de Arte Moderno), 1994.

2. Pour une explication de la signification cinématographique de l'œuvre de Jeff Wall, voir C. Dercon (2000: 117-128). Pour la définition, la validité et la complexité des structures narratives dans la photographie contemporaine, on peut consulter L. Soutter (2000: 9-12).

3. L'Iconología (1593) de Cesare Ripa, sorte d'encyclopédie des allégories et des symboles du XVI e siècle, était une source d'une importance capitale pour l'iconographie catholique pendant les XVII et XVIII' siècles.

4. L'artiste tient dans ses bras l'acteur Robert Downey. D'après la sculpture de Michel-Ange, La Pietà, 1499.

5. D'après un tableau anonyme de l'École de Fontainebleau, fin du $\mathrm{XVI} \mathrm{I}^{\mathrm{e}}$ siècle (Gabrielle d'Estrées et une de ses soeurs).

6. En ce qui concerne l'espace de la figuration, le mode théâtral et le renvoi à des faits documentaires dans les tableaux photographiques de Wall, voir Chevrier (1995: 11-16).

7. Pour une discussion des définitions glissantes des termes "photographe commercial» et "photographe d'art", voir A. Sekula (1990: 39-45). 


\section{RÉFÉREN CES BIBLIO G RAPHIQ U ES}

AtKinson, T. [1993] : "Dead Troops Talk", Jeff Wall. Dead Troops Talk, Lucerne, Kunstmuseum Luzern, 29-45.

BARTHES, R. [1977]: "The Third Meaning: Notes on Some of

Eisenstein's Stills ", Image-Music-Text, New York (publication originale dans Artforum, janvier 1973).

BOECKER, S. [2001] : "Bernhard Prinz», Kunstforum, nº 154 (avril-mai), 414-415.

ChEVRIER, J. F. [1995] : "Play, Drama, Enigma », Jeff Wall, Paris, Galerie Nationale du Jeu de Paume, 11-16.

Clark, R. [2001]: "John Goto. High Summer", Portfolio. The Catalogue of Contemporary Photography in Britain, no 33, 4-11.

DERCON, C. [2000] : "Jeff Wall. De beeldende kunst imiteert de film ",

Ik zou een museum willen maken waar de dingen elkaar overlappen,

Rotterdam, 117-128.

Folie, S. et M. Glasmeier [2002] : «Atmende Bilder. Tableau vivant und Attitüde zwichen "Wirklichkeit and Imagination" ", Tableaux

Vivants. Lebende Bilder und Attituden in Fotografie, Film und Video,

Vienne, Kunsthalle Wien, 9-52.

FRIED, M. [1981]: «Representing Representation: On the Central Group in Courbet's Studio ", dans S.J. Greenblatt (dir.), Allegory and Representation. Selected Papers from the English Institute, Baltimore et Londres, 94-127.

GreEnblatT, S.J. [1981]: "Preface», Allegory and Representation, 1979. 80, Baltimore et Londres, VII-XIII.

HOLBORN, M. [1987]: Opera on a Grand Scale. David Buckland, Londres, The Photographers' Gallery.

Lowry, J. [2002]: "Melanie Manchot. A Gestural Politics of the Real", Portfolio. The Catalogue of Contemporary Photography in Britain, $\mathrm{n}^{\circ} 35,54$ 55
MCGuire, M. [1997]: "Virtue and Vice», Variant, vol. 2, no3 (été), 2-3. OwENS, C. [1984]: "The Allegorical Impulse: Toward a Theory of Postmodernism ", dans B. Wallis et M. Tucker (dir.), Art After Modernism: Rethinking Representation, New York, New Museum of Contemporary Art, 203-235 (éd. originale dans October, n 12 , 67-86 et no $13,59-80,1980$ )

PORCHERON, M.D. [2000]: Dany Leriche. Les Filles de Ripa, préface au catalogue, Paris, Galerie Rachlin-Lemarié, 3-7.

RamíreZ, J.A. [1994] : "Portraits, Allegories, Mirrors... ", Los Géneros de la Pintura. Una visión actual, Las Palmas (Grande Canarie), Centro Atlantico de Arte Moderno, 171-175.

SANTACATTERINA, S. [2002]: "The Super Art of Sam Taylor-Wood ", Portfolio. The Catalogue of Contemporary Photography in Britain, no35, 52 53.

Sekula, A. [1990]: "Some American Notes", Art in America, février, 39-45.

SlyCE, J. [1999]: «On Time, Narration and Performative Realism. The Photographs of Sarah Jones ", Sarah Jones, Essen, Folkwang Museum, 101-119.

SOUTTER, L. [2000] : "Dial "P” for Panties : Narrative Photography in the 1990s ", Afterimage, janvier.

THIJSEN, M. [1997]: "The Aim of Allegory. Somewhere between form and content", Virtue $\mathcal{E}$ Vice. Derivations of Allegory in Contemporary Photography, Sheffield, Site Gallery, 6-22;

- [1999]: «El propósito de la alegoría: entre la forma y el contenido ", Papel Alpha, n4, 83-101.

Wallis, B. [1987]: Blasted Allegories. An Anthology of Writings by Contemporary Artists (Documentary sources in Contemporary Art), vol. 2, Cambridge. 